\title{
An Alternative Method of Stochastic Optimization: The Portfolio Model
}

\author{
Moawia Alghalith \\ University of the West India, Saint Augustine, Trinidad and Tobago \\ E-mail: malghalith@gmail.com \\ Received May 19, 2011; revised May 26, 2011; accepted May 29, 2011
}

\begin{abstract}
We provide a new simple approach to stochastic dynamic optimization. In doing so, we derive the existing (standard) results using a far simpler technique than the duality and the variational methods.
\end{abstract}

Keywords: Stochastic Optimization, Investment, Portfolio

\section{Introduction}

Previous studies in stochastic optimization relied on the duality approach and/or variational techniques such as using the Feynman Kac formula and the HamiltonJacobi-Bellman partial differential equations. Examples include [1-3], among many others.

In this paper, we offer a new simple approach to stochastic dynamic optimization. That is, we prove the previous results using a simpler method than the duality or the Hamilton-Jacobi-Bellman partial differential equations methods. We apply our method to the standard investment model. Our approach is based on dividing the time horizon into sub-horizons and applying Stein's lemma.

\section{The Portfolio Model}

We use the standard investment model (see, for example, [3], among many others). Similar to previous models, we consider a risky asset and a risk-free asset. The risk-free asset price process is given by $S_{0}=e^{\int^{t} r d s}$, where $r \in C_{b}^{2}(R)$ is the rate of return.

The dynamics of the risky asset price are given by

$$
\mathrm{d} S_{s}=S_{s}\left\{\mu \mathrm{d} s+\sigma \mathrm{d} W_{s}\right\},
$$

where $\mu$ and $\sigma$ are the deterministic rate of return and the volatility, respectively, and $W_{s}$ is a standard Brownian motion.

The wealth process is given by

$$
X_{T}^{\pi}=x+\int_{t}^{T}\left\{r X_{s}^{\pi}+\left(\mu_{s}-r_{s}\right) \pi_{s}\right\} \mathrm{d} s+\int_{t}^{T} \pi_{s} \sigma_{s} \mathrm{~d} W_{s},
$$

where $x$ is the initial wealth, $\left\{\pi_{s}\right\}_{t \leq s \leq T}$ is the risky portfolio process with $E \int_{t}^{T} \pi_{s}^{2} \mathrm{~d} s<\infty$. The trading strategy $\pi_{s} \in \mathcal{A}(x)$ is admissible (that is, $X_{s}^{\pi} \geq 0$ ).

The investor's objective is to maximize the expected utility of the terminal wealth

$$
V(t, x)=\operatorname{Sup}_{\pi} E\left[U\left(X_{T}^{\pi}\right) \mid \mathcal{F}_{t}\right]=E\left[U\left(\pi^{*}\right) \mid \mathcal{F}_{t}\right],
$$

where $V($.$) is the (smooth) value function, U($.$) is$ continuous, bounded and strictly concave utility function, and $\mathcal{F}$ is the filtration.

We rewrite (2) as

$$
\begin{aligned}
X_{T}^{\pi}= & x+r_{u} X_{u}^{\pi}+\left(\mu_{u}-r_{u}\right) \pi_{u}+\pi_{u} \sigma_{u} W_{u} \\
& +\int_{\bar{t}}^{T}\left\{r_{s} X_{s}^{\pi}+\left(\mu_{s}-r_{s}\right) \pi_{s}\right\} \mathrm{d} s+\int_{\bar{t}}^{T} \pi_{s} \sigma_{s} \mathrm{~d} W_{s} \\
& +\int_{t}^{T}\left\{r_{s} X_{s}^{\pi}+\left(\mu_{s}-r_{s}\right) \pi_{s}\right\} \mathrm{d} s+\int_{t}^{T} \pi_{s} \sigma_{s} \mathrm{~d} W_{s} ; \\
\bar{t}< & u<t, u \notin[\bar{t}, T] \cup[t, T], u \in[t, T] .
\end{aligned}
$$

Substituting the above equation into (3) and differentiating with respect to $\pi_{u}^{*}$ (and setting the derivative equal to zero) yields

$$
\left(\mu_{u}-r_{u}\right) E\left[U^{\prime}(.) \mid \mathcal{F}_{t}\right]+\sigma_{u} E\left[U^{\prime}(.) W_{u} \mid \mathcal{F}_{t}\right]=0 .
$$

By Stein's lemma

$$
\begin{aligned}
E\left[U^{\prime}(.) W_{u} \mid \mathcal{F}_{t}\right] & =\operatorname{Cov}\left(X_{u}, W_{u}\right) E\left[U^{\prime \prime}(.) \mid \mathcal{F}_{t}\right] \\
& =\pi_{u}^{*} \sigma_{u} E\left[U^{\prime \prime}(.) \mid \mathcal{F}_{t}\right] .
\end{aligned}
$$


Substituting this into (5) yields

$$
\pi_{u}^{*}=-\frac{\left(\mu_{u}-r_{u}\right) E\left[U^{\prime}(.) \mid \mathcal{F}_{t}\right]}{\sigma_{u}^{2} E\left[U^{\prime \prime}(.) \mid \mathcal{F}_{t}\right]}=-\frac{\left(\mu_{u}-r_{u}\right) V_{x}(.)}{\sigma_{u}^{2} V_{x x}(.)} .
$$

This solution can be generalized to any point on time $s$

$$
\pi_{s}^{*}=-\frac{\left(\mu_{s}-r_{s}\right) V_{x}(.)}{\sigma_{s}^{2} V_{x x}(.)}
$$

This is exactly the solution obtained by the previous literature, but its derivation is far simpler. Furthermore, this approach can be applied to many other stochastic models.

\section{References}

[1] M. Alghalith, "A New Stochastic Factor Model: General Explicit Solutions," Applied Mathematics Letters, Vol. 22, No. 12, 2009, pp. 1852-1854. doi:10.1016/j.aml.2009.07.011

[2] W. Fleming, "Some Optimal Investment, Production and Consumption Models," Contemporary Mathematics: Mathematics of Finance AMS-IMS-SIAM Proceedings, 2004, pp. 115-124.

[3] F. Focardi and F. Fabozzi, "The Mathematics of Financial Modeling and Investment Management," Wiley, Hoboken, 2004. 